\title{
Abnormal Signatures Recorded by FORMOSAT-2 and FORMOSAT-3 over South Atlantic Anomaly and Polar Region
}

\author{
Tsung-Ping Lee ${ }^{1}$, Panthalingal Krishnanunni Rajesh ${ }^{2}$, Chao-Yen Chen ${ }^{2}$, Jann-Yenq Liu ${ }^{1,2,3, *}$, \\ Chen-Joe Fong ${ }^{1}$, Jar-Chen Pon ${ }^{1}$, Shan-Kuo Yang ${ }^{1}$, and Guey-Shin Chang ${ }^{1}$ \\ ${ }^{1}$ National Space Organization, HsinChu, Taiwan \\ ${ }^{2}$ Institute of Space Science, National Central University, Jhongli City, Taiwan \\ ${ }^{3}$ Center for Space and Remote Sensing Research, National Central University, Jhongli City, Taiwan
}

Received 13 June 2013, revised 25 February 2014, accepted 26 February 2014

\begin{abstract}
Computer systems onboard FORMOSAT-2 (F2) and FORMOSAT-3/COSMIC (F3/C) satellites often register abnormal signatures which are recorded as automatic reconfiguration orders (ARO) in F2, and reboot/reset (RBS) in F3/C. The ARO and RBS spatial distribution counts recorded since the launch of satellites is investigated to identify regions of anomalous events. Data from the star tracker onboard F2 and Tiny Ionosphere Photometer (TIP) onboard F3/C are also analyzed. The results show that the F2 ARO and F3/C RBS cluster over the SAA (South Atlantic Anomaly) region and also over the poles, which suggest that high energy particles bombarding the satellite electronics play an important role.
\end{abstract}

Key words: South Atlantic Anomaly (SAA), Single Event Upset (SEU), FORMOSAT-2, FORMOSAT-3

Citation:Lee, T.P., P. K.Rajesh, C.Y.Chen, J.Y.Liu, C.J.Fong, J.C.Pon, S. K.Yang, and G.S.Chang, 2014: Abnormal signatures recorded by FORMOSAT-2 and FORMOSAT-3 over South Atlantic Anomaly and Polar Region. Terr. Atmos. Ocean. Sci., 25, 573-580, doi: 10.3319/TAO.2014.02.26.01(AA)

\section{INTRODUCTION}

In the space environment satellite electronics and onboard computer systems are susceptible to interactions with energetic particles, which could result in the generation of anomalous signals that affect the measurements. A flux of protons with energy over $1 \mathrm{MeV}$ or electrons with energy of $0.5 \mathrm{MeV}$ may cause sensitive electronic parts to malfunction (Gubby and Evans 2002). However, if the proton (electron) energy exceeds $10 \mathrm{MeV}(2 \mathrm{MeV})$ the effect could be very severe, resulting in the failure of on-board semi-conductor devices, known as Single Event Latch-up, or signal inversion, usually referred to as Single Event Upset (SEU) (Guenzer et al. 1979; Kolasinski et al. 1979; Bashkirov et al. 1999). SEU is a change of state caused by ions or electromagnetic radiation striking a sensitive node in a microelectronic device, such as a sensitive region of a transistor in flip-flop circuit (Baker 2000), usually defined as bits flip and might cause the onboard-computer to reboot or reset.

Such disruption of onboard electronics by energetic particles has been a matter of concern for scientists and en-

\footnotetext{
* Corresponding author

E-mail: tiger@nspo.narl.org.tw
}

gineers over several decades, and is often reported over the South Atlantic Anomaly (SAA) and polar region (Reed et al. 1973; Torr et al. 1975; Gledhill 1976; Campbell et al. 1992; Fleetwood et al. 2000). SAA, a region of low magnetic field in the South Atlantic Ocean extending over to the South American continent is generated due to the offset of the Earth's magnetic dipole and is a major sink of trapped charged particles, giving rise to electron and proton precipitation (Dessler 1959). In this study, a total magnetic field strength of $22700 \mathrm{nT}$ is used as the threshold to define the SAA region. The presence of energetic particles over the region in the $320 \pm 45 \mathrm{~km}$ altitude range was initially detected by one of the COSMOS satellites (Vernov et al. 1967). On the other hand the magnetic field geometry renders the polar ionosphere accessible to energetic particles and is a major source of ionization in the high latitudes (Sergeev et al. 1983; Lyons 1997). Several studies have shown that galactic cosmic rays, solar energetic particles, trapped high energetic particles and electrons contribute to SEU or cause significant problems to satellite systems (Bashkirov et al. 1999; Baker 2000; Gubby and Evans 2002; Nichitiu et al. 2004; Koshiishi et al. 2008; Patil et al. 2008; Kuznetsov 
et al. 2010). During high solar activity periods the impact of space weather events could cause more frequent system failures (Nichitiu et al. 2004; Iucci et al. 2006).

This work focuses on the odds of satellite reset occurrence on FORMOSAT-2 (F2) and FORMOSAT-3/COSMIC (F3/C) Taiwan missions over the SAA and polar region in comparison to other locations, using data from the Taiwan Analysis Center for COSMIC and the quality information system of the National Space Organization (NSPO), where COSMIC stands for Constellation Observing System for Meteorology, Ionosphere, and Climate. The F2 electronic anomaly is known as automatic reconfiguration order (ARO), and in the case of $\mathrm{F} 3 / \mathrm{C}$, the interruptions cause the reboot/reset (RBS) of the onboard computer. After the launch of F2, there have been about 13 ARO events identified as SEU, whereas in the case F3/C about 385 RBS events have been recorded till 31 May 2011. This enormous number of reset events is the main motivation for setting up a task-force team to investigate the cause of SEUs in space. In this study the global distribution of the locations of F2 ARO and $\mathrm{F} 3 / \mathrm{C}$ RBS occurrences is investigated and the influence of such events over the SAA region on satellite missions is discussed. Star tracker data in F2 as well as airglow observations by Tiny Ionosphere Photometer (TIP) onboard F3/C are used to analyze the anomaly distribution experienced by the satellite sensor and payload. The possible reasons for the observed difference in the number of anomalies in F2 and F3/C are discussed, which could be important considering that follow-up mission for $\mathrm{F} 3 / \mathrm{C}$ is currently being planned with more satellites as part of the constellation.

\section{SEU EVENTS ON F2 AND F3/C}

$\mathrm{F} 2$ is the remote sensing space program of NSPO, Taiwan, launched on 21 May 2004, to an initial orbit at $723 \mathrm{~km}$. The final mission orbit is at $891 \mathrm{~km}$ with an inclination angle of $99^{\circ}$. The primary goals of the F2 mission are remote sensing applications for natural disaster evaluation, environmental monitoring and ocean surveillance. The anomaly of the F2 electronics, known as ARO, is a computer reset mechanism that protects the satellite from any permanent damage but will disturb the satellite systems for at least one day. When two consecutive cyclic tasks cannot be accomplished within a pre-allocated time the flight software will issue an error message 'overload', which will trigger an ARO. The analysis carried out for this work further revealed that in addition to the energetic particles, star tracker lost bytes (LB) events could also contribute to ARO. An LB event occurs when the telemetry data from the star tracker to the on-board computer fails in the cyclic redundancy check and is discarded by the flight software. Out of the 34 AROs that occurred on F2 until 31 May 2011, 13 events are designated as SEU.

The F3/C mission, consisting of six Low-Earth-Orbit satellites (Rocken et al. 2000; Fong et al. 2009), is the first constellation demonstrating near real-time Numerical Weather Prediction, and makes use of occultation of radio signals from the Global Positioning System (GPS) satellites (Liou et al. 2007). F3/C was launched on 15 April 2006 to an initial orbit of about $516 \mathrm{~km}$. The satellites were subsequently lifted to the mission orbit at $800 \mathrm{~km}$, with an inclination of $72^{\circ}$. On F3/C, the RBS anomaly events could be caused by the interruption of attitude control electronic computer, or battery control regulator computer, or flight computer. The satellite design has been optimized to shorten the recovery time from different types of resets. So far about 385 F3/C computer anomalies have been designated as SEUs.

The computer reboot or reset may occur due to several factors, and identifying which of them could be caused by SEU involves detailed analysis of various system health messages. The reboot or reset could be triggered due to some error in the flight software, telemetry glitches, power problems, or any hardware issues, in addition to that by SEU. In F3/C, when a computer RBS event is identified, several post-investigations are carried out to ensure that it was not caused by a power code reset, link failure, reset due to power shortage, telemetry glitch, or software reboot. All of the system messages are carefully analyzed and if all such causes are completely ruled out, then such an event is considered as produced by SEU. Similarly, in F2, there is a dedicated application ID for different kinds of such errors and after confirming that the error was not caused by any such factors the SEU can be recognized from the error ID reported.

\section{RESULT AND INTERPRETATION}

A total of 34 ARO events occurred on the F2 between 21 May 2004 and 31 May 2011, out of which 13 were due to SEUs. Figure 1 gives the global distribution of F2 ARO events. Note that about 15 AROs were recorded over the SAA and polar region and 7 of them were SEUs, which is more than $50 \%$ of the SEU events. Among the $15 \mathrm{SAA} / \mathrm{po}-$ lar events, about 6 AROs were registered within and nearby SAA region, which include 4 events attributed to SEU.

As mentioned earlier, since LB events also trigger ARO, the distribution of LB was analyzed to confirm if it contributed to the ARO occurrence. An LB is reported when the star tracker ephemeris data to the flight software cannot be identified within a scheduled time interval, and several such LB events occurring successively will result in an error message 'overrun'. Two consecutive overruns will trigger an overload message and ARO will follow. In addition to LB, if the star tracker data header is contaminated, the flight software will attempt to re-analyze the header information to find the actual data. However, if the data is severely contaminated to complete the analysis within a specific time interval, an overrun message will be issued, and could lead to an ARO. During the 2566 days of observations since its launch, the F2 generated $2298 \mathrm{LB}$ events and 43 overruns. Figure 2 illustrates the 
LB distribution along the satellite orbits. It can be seen that the LBs occur more or less uniformly along the tracks and there is no apparent increase over SAA or the poles.

In order to further investigate the occurrence of ARO and LB over high energy particle precipitation locations the latitudinal distribution of the events in $10^{\circ}$ bins over the entire longitudinal range is computed. Since the surface area of the longitude slices in different latitude bins are different, the total counts of ARO and LB in each bin are normalized using the ratio of the surface area of the corresponding bin to that of the bin having the lowest area $\left(80^{\circ}-90^{\circ}\right)$, with the results plotted in Fig. 3. The latitudinal distribution reveals that ARO and LB events occur more over high latitude regions and decrease towards the equator. Further, it can be seen that over the SAA latitude $\left(20^{\circ}-30^{\circ} \mathrm{S}\right)$ there is an increase in ARO, but it is absent in the case of LB.
On F3/C, there have been about 385 computer RBSs, out of which, 274 events occurred over the SAA and Polar Regions. The distribution of F3/C computer reset locations is given in Fig. 4. It can be seen that the occurrence density of the reset events is higher over the SAA region than other locations. The reboot/reset (RBS) events over SAA and polar region are over $70 \%$ of the total occurrence.

The OI $135.6 \mathrm{~nm}$ airglow intensity distribution observed by TIP onboard F3/C is also analyzed to understand the influence of energetic particles on the measurements. The TIP is a nadir viewing compact, high sensitive UV photometer onboard the F3/C satellites that measures the integrated OI $135.6 \mathrm{~nm}$ intensity along the line-of-sight (Coker et al. 2009; Hsu et al. 2009). TIP can detect even minute intensity variations with a very high sensitivity of about 500 counts second ${ }^{-1}$ Rayleigh $^{-1}$. It has a field of view of $3.8^{\circ}$,

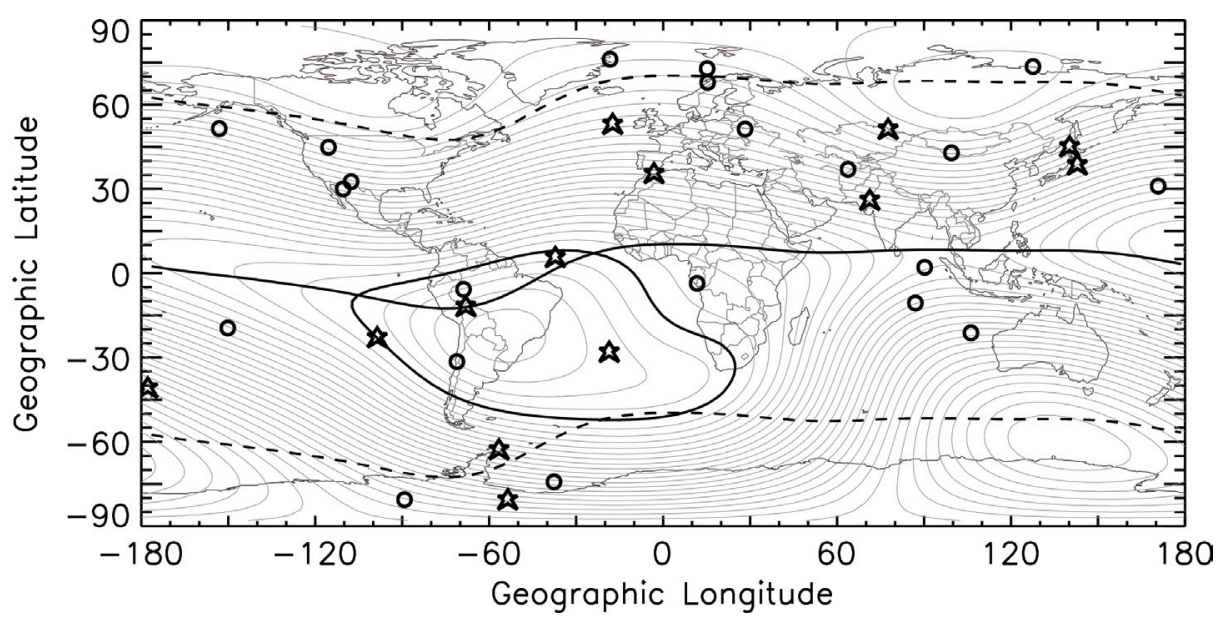

Fig. 1.F2 ARO distributions during 21 May 2004 to 31 May 2011. The asterisk symbols denote the ARO related to SEU events and the open circles represent other types of ARO. The black solid line is the dip equator, and the dashed lines show locations $60^{\circ}$ off the equator in both the hemispheres. The thin black contours are the total field intensity using IGRF 2011 coefficients. The thick black contour line corresponding to a magnetic field strength of $22700 \mathrm{nT}$, highlights the SAA region.

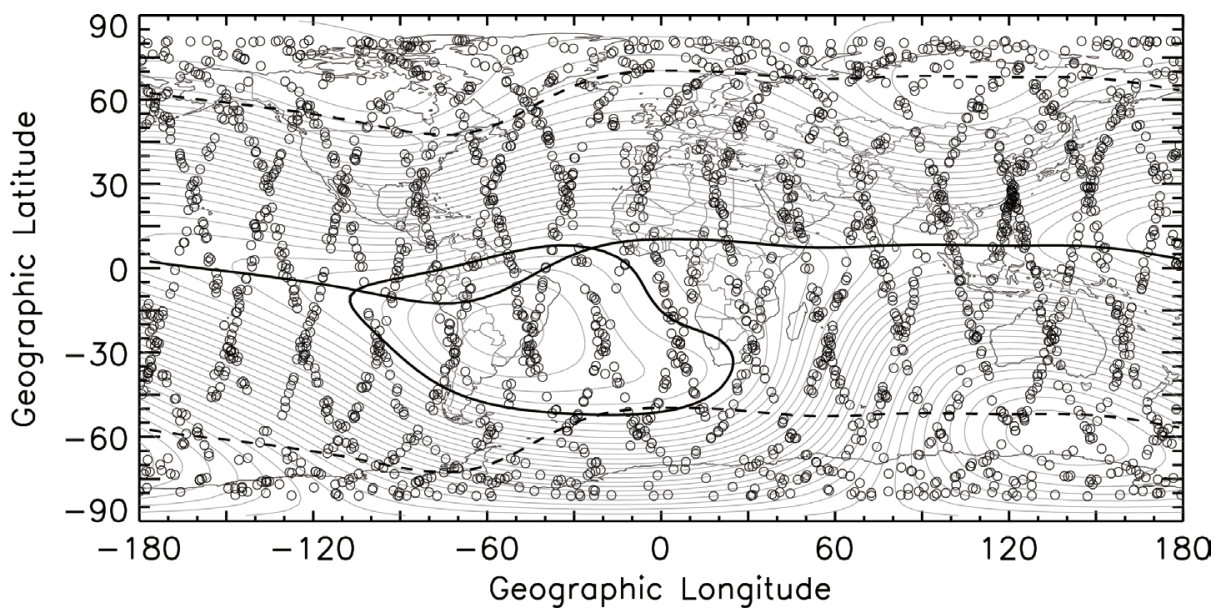

Fig. 2. The F2 LB distribution during 21 May 2004 to 31 May 2011. The open circles denote the location of LB occurrence. The other curves are similar to that in Fig. 1. 


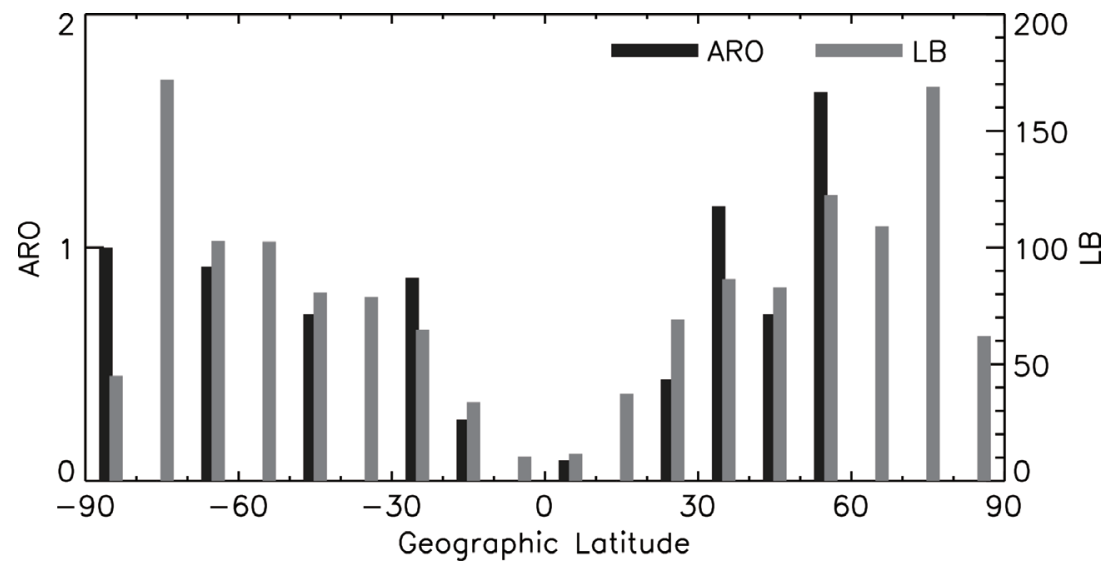

Fig. 3. Latitudinal distribution of F2 ARO and LB occurrence. The total occurrence in each $10^{\circ}$ latitude bin is normalized using the ratio of the surface area of the corresponding bin to that of the bin having the lowest area.

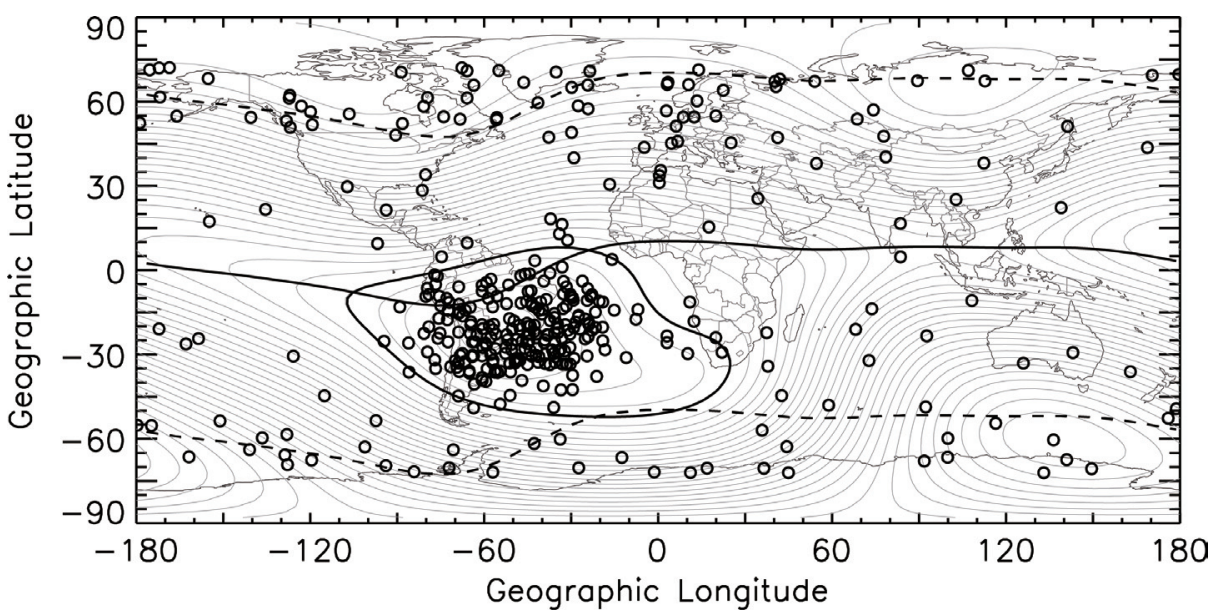

Fig. 4. F3/C RBS distributions between 15 April 2006 and 31 December 2010. Open circles denote RBS events. Other curves have the similar meaning as in Fig. 1.

which corresponds to a spatial resolution of about $30 \mathrm{~km}$ at a height of $350 \mathrm{~km}$ (altitude of peak emission), from the satellite altitude of $\sim 800 \mathrm{~km}$. Figure 5 displays the OI $135.6 \mathrm{~nm}$ intensity distribution from TIP during 2006 - 2010. The excitation cross-section of the production of $\mathrm{O}\left({ }^{5} \mathrm{~S}\right)$ state that gives the OI $135.6 \mathrm{~nm}$ emission peaks in the energy range of a few tens of $\mathrm{eV}$ to $100 \mathrm{eV}$. It can be seen from the figure that over the SAA and Polar Regions the airglow intensity is significantly greater than at other locations. The stronger intensity over the SAA region agrees well with the distribution of the reset events in Fig. 4.

As in the case of F2, a similar analysis was carried out to estimate the latitudinal distribution of the reset counts in F3/C. The result is displayed in Fig. 6, together with the corresponding TIP intensity. It is evident that a significant number of satellite reset events were recorded over the SAA and polar region. The TIP intensity also exhibits a similar latitude variation. Compared with F2, there are many more enhanced onboard computer reset events for the case of $\mathrm{F} 3 / \mathrm{C}$ over
SAA. Figure 7 gives the longitude distribution of F2 ARO and F3/C RBS events. It can be seen that the peak between $30^{\circ}-60^{\circ} \mathrm{W}$ longitudes corresponds to the SAA region. Note that data from all six F3/C satellites were used in this study.

\section{DISCUSSION AND CONCLUSION}

The results described here show that the occurrence of F2 ARO events and F3/C resets are pronounced over the SAA and polar region, indicating that SEU events related to energetic particles significantly affect satellite performance. The latitudinal distribution suggests that F2 ARO peaks over SAA and the star tracker LB do not. LB could occur if the star tracker CCD is directly affected by particles rendering the image unidentifiable with respect to reference data and also if the CCD image is contaminated by background luminosity. The enhanced LB occurrence over the Poles comes from the extended coverage of the mission orbits in that region, involving 14 daily revisits. In the absence of any known 


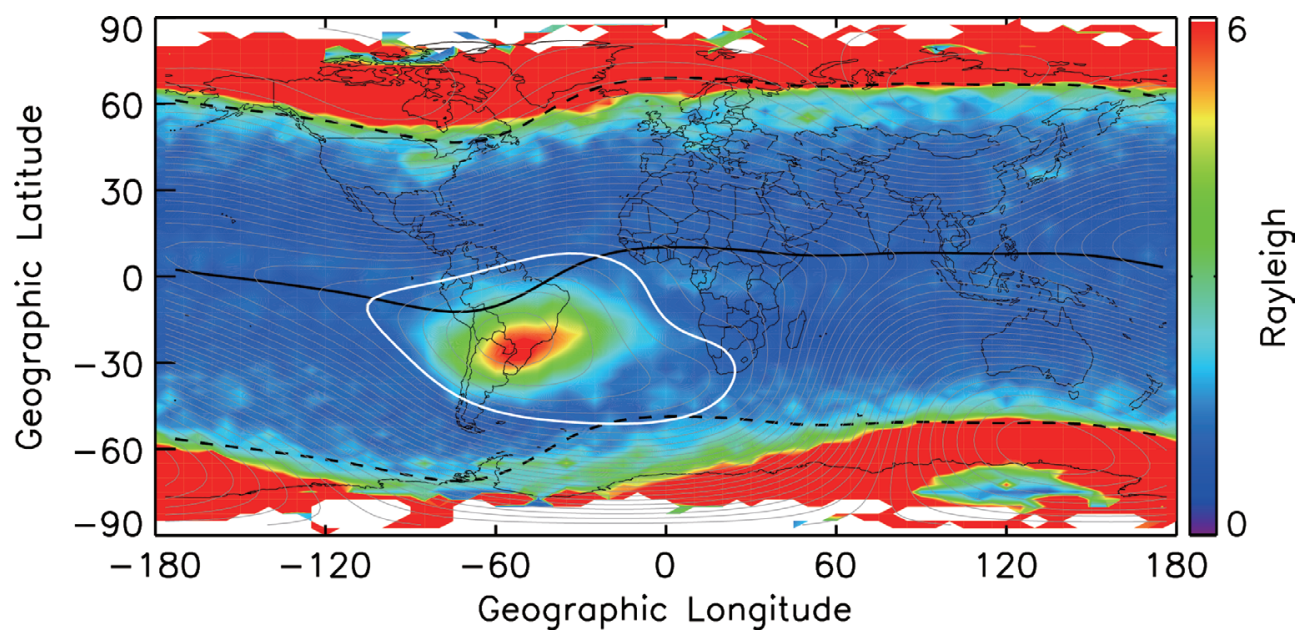

Fig. 5. The OI $135.6 \mathrm{~nm}$ airglow intensity distribution observed by TIP during 2006 - 2010. Other curves have the similar meaning as in Fig. 1.

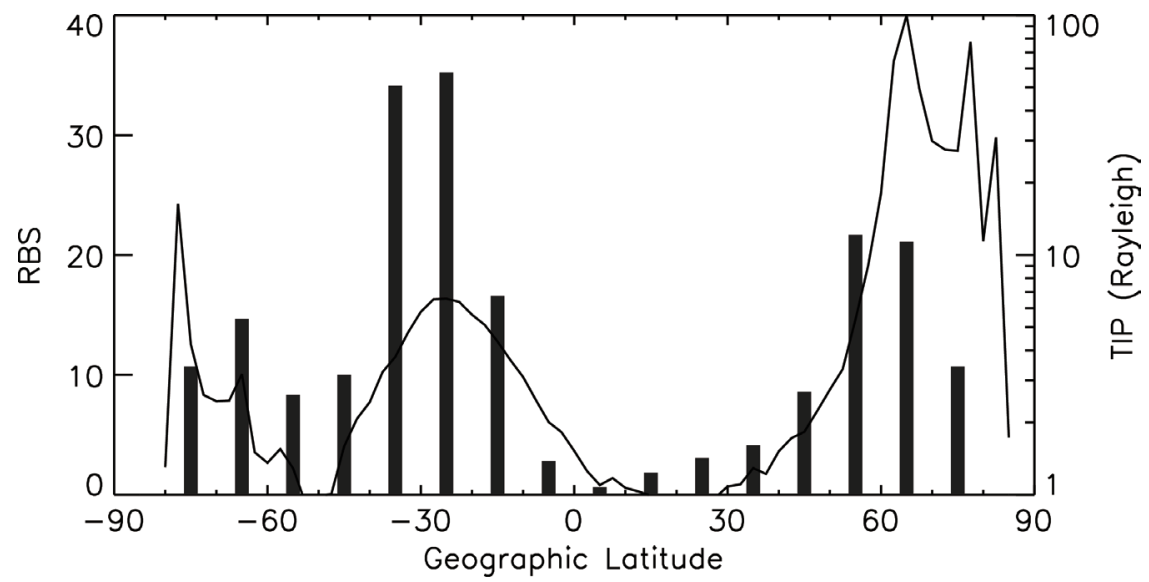

Fig. 6. Latitudinal distributions of F3/C RBS and TIP intensity. The histograms show the RBS occurrence and the back curve is the average TIP intensity over $45^{\circ}-55^{\circ} \mathrm{W}$ longitude.

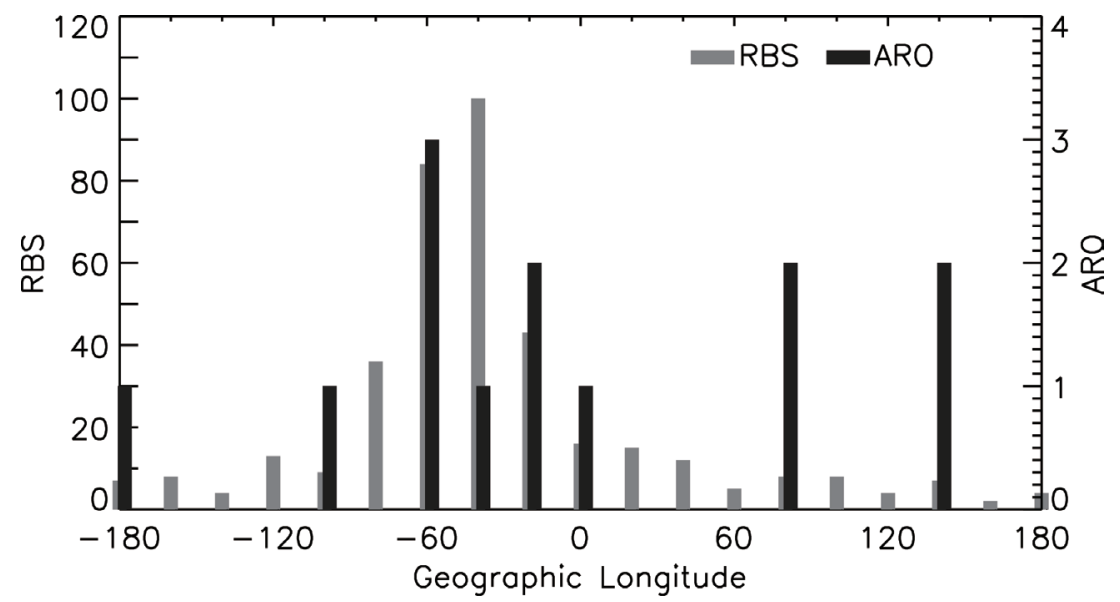

Fig. 7. Longitude distributions of F3/C RBS and F2 ARO events. 
sources to trigger SEU, the observed AROs around $30^{\circ} \mathrm{N}$ could be speculated to be related to LB events. When several LB are reported successively, the same overload signal is triggered resulting from a SEU event, causing an ARO.

In F3/C, the TIP observations show pronounced intensity over the SAA region, coinciding with the enhanced reset counts, indicating that energetic particles affect the airglow measurement. Figure 8 gives the TIP intensities measured over the SAA region during 2006 - 2010 as a function of the corresponding F3/C altitude. The satellites were first launched to the parking orbit around $500 \mathrm{~km}$, and were gradually lifted to the mission orbit at around $800 \mathrm{~km}$, taking a period of more than a year for the process. It can be seen from Fig. 8 that the TIP intensities measured at the two altitude levels are different, with lesser values when the satellite altitude is below $600 \mathrm{~km}$. This suggests that the energetic particles could possibly contaminate the detector and thus the measurements when the satellite altitude is greater and the effect is not severe if the satellite is placed at a slightly lower orbit.

However, the strong enhancement in the TIP intensity over the SAA region could come from the energetic particle effect as well as the actual airglow intensity over the region through photochemical processes and collisional excitation. The energetic particles could cause malfunctioning of the electronics and the photometer itself could also be affected. In addition, the particle precipitation could also enhance the airglow emission by populating the excited levels of atomic oxygen, with the combined effect giving the strong intensity over SAA. Different types of energetic particle precipitation effects in the ionosphere and thermosphere over the SAA have been discussed in the literature (see for example, Abdu et al. 2005). Since TIP makes nadir integrated measurements, the effect of the two could not be distinguished in the data. On the other hand, the Imager of Sprites and Upper Atmo- spheric Lightnings (ISUAL) on F2, which takes limb images of $630.0 \mathrm{~nm}$ emissions, indeed observe intense airglow regions over the SAA. Figure 9 gives an example of ISUAL observation from a track, which is away from the SAA region but the line-of-sights of the imager collect the emission including the SAA region. Observations from this track will hence give the airglow signature without any contamination to the detector itself. Note that the ISUAL observations include an upper $\mathrm{O}\left({ }^{1} \mathrm{D}\right)$ layer and a lower $\mathrm{OH}(9$ - 3) layer in the limb images (Rajesh et al. 2009; Nee et al. 2010). The strong $\mathrm{O}\left({ }^{\prime} \mathrm{D}\right)$ enhancement between $30^{\circ}-35^{\circ} \mathrm{S}$ latitudes, occurring below the tangent altitude of $200 \mathrm{~km}$, most likely results from the energetic particle contribution to the emission. There is no other known excitation mechanism to account for the observed enhancement at these latitudes. Further, the relatively lower altitude of the peak intensity also points to the energy transfer by precipitating low energy particles.

The results described here also show that the number of $\mathrm{F} 3 / \mathrm{C}$ resets over the SAA region is much greater than the corresponding F2 ARO events. Note that the F2 is shielded well with Aluminum and employs highly reliable electrical, electronic and electromechanical parts, whereas the $\mathrm{F} 3 / \mathrm{C}$ does not. This could be one of the reasons for more resets occurred in F3/C. Moreover, since the inclination of F2 $\left(99^{\circ}\right)$ is greater than that of $\mathrm{F} 3 / \mathrm{C}\left(72^{\circ}\right)$, the $\mathrm{F} 2$ distributions peak over the polar region, whereas the $\mathrm{F} 3 / \mathrm{C}$ resets are mainly over SAA (Iucci et al. 2006). The analysis further reveals that the odds of both F2 ARO and F3/C reset over the SAA region compared to other locations are greater than 2.5, which implies both are statistically significant as shown by Liu et al. (2006) while analyzing pre-earthquake ionospheric signatures. This again indicates that the SAA region could have severe impact that affects satellite systems.

In conclusion, the results suggest that energetic particles

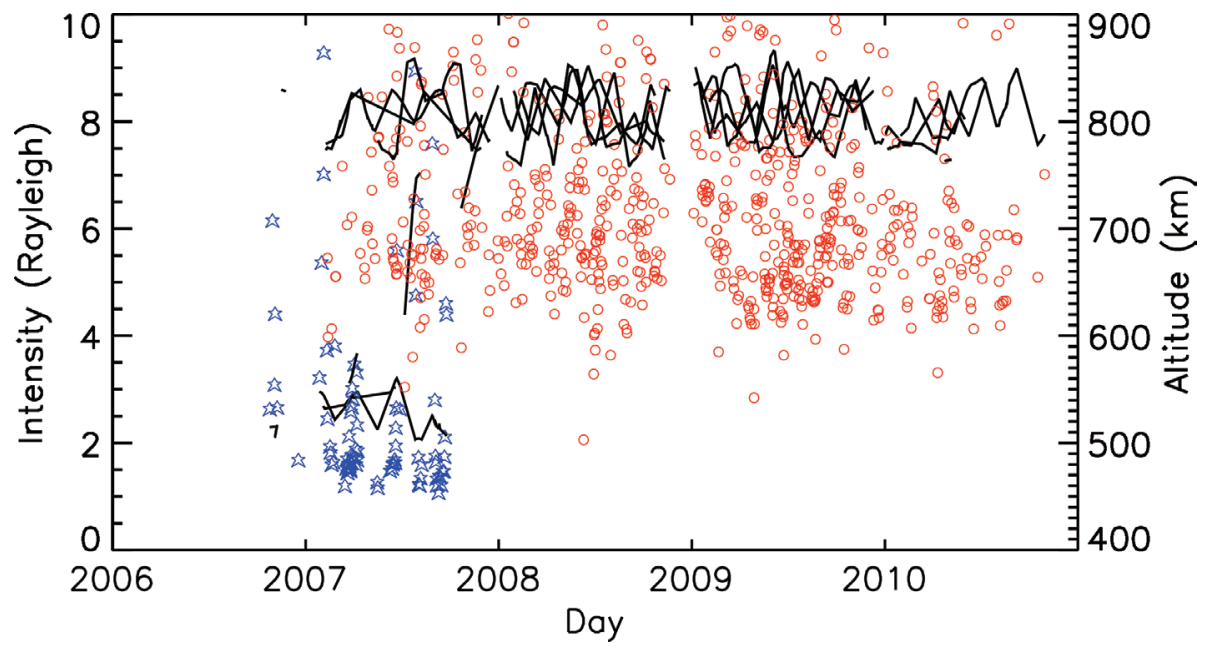

Fig. 8. The TIP intensity over SAA at different F3/C altitudes as a function of days after the launch. The blue asterisks denote the TIP intensity when the satellite altitude is below $600 \mathrm{~km}$, and the red circles indicate the intensity when the satellite altitude is above $600 \mathrm{~km}$. The dark lines denote the satellite altitude. The data taken during 1 hour interval centered at $2200 \mathrm{LT}$, within a $\pm 5^{\circ}$ grid over $50^{\circ} \mathrm{W}$ and $25^{\circ} \mathrm{S}$, are used. 

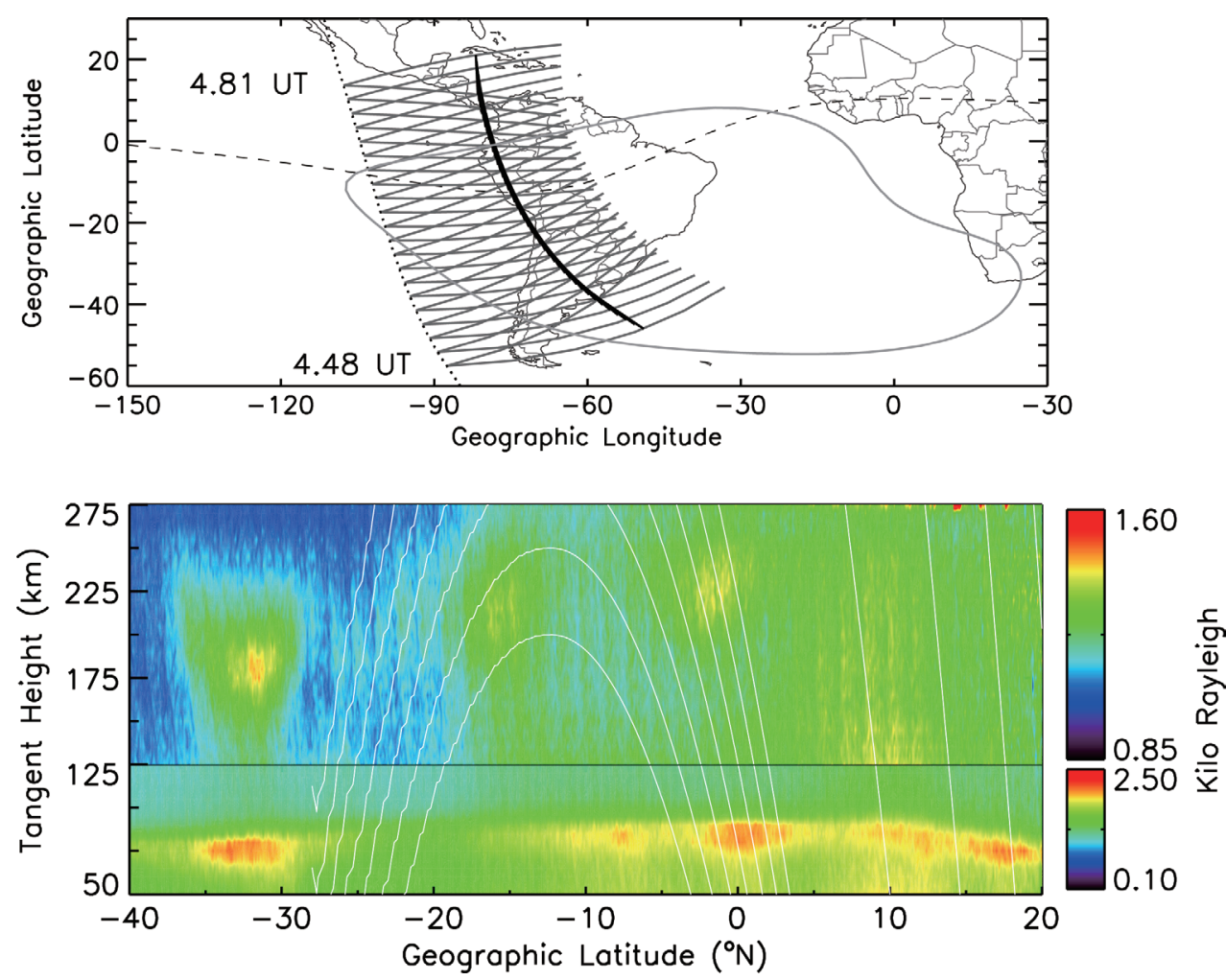

Fig. 9. Airglow enhancement over the SAA region in ISUAL 630.0 limb images. The satellite track (dotted curve), line-of-sights corresponding to the field-of-view (gray lines starting at the orbit), and the tangent plane (dark curve parallel to the track) are indicated in the top panel. Note that since the limb integrated emission is plotted at the tangent coordinates, the altitude of the layer appears about $20-25 \mathrm{~km}$ lower than the true altitude. The satellite orbits away from the SAA region, while the line-of-sight collects emissions over the SAA. The start and end time of the observations are marked. The dashed line denotes the magnetic equator and the gray contour is the total field intensity representing the SAA coverage. The bottom panel gives the limb image at $630.0 \mathrm{~nm}$ observed by ISUAL on 17 July 2008 over the track. The strong enhancement over $30^{\circ}-35^{\circ} \mathrm{S}$ corresponds to the SAA location, and the altitude of emission is lower than the altitude of the layer at other latitudes. Note that two different color bars are used to give better contrast for the intensities of the two emission layers: the upper $\left[\mathrm{O}\left({ }^{1} \mathrm{D}\right)\right]$ centered at $630.0 \mathrm{~nm}$ and the lower $[\mathrm{OH}(9-3)]$ in the wavelength range of $625.0-631.0 \mathrm{~nm}$.

over the SAA region significantly influence satellite performance, triggering SEU events and also probably contaminating optical measurements. However, the effect could be minimized in the forthcoming satellite missions by making suitable orbit adjustments, using proper aluminum shielding and with the help of more robust and reliable design and component selection. Further, TIP serves as a detector of particle precipitation over SAA region, while ISUAL could monitor the airglow enhancement by the energetic particles.

Acknowledgment The authors would like to thank NSPO Satellite Operations Control Center (SOCC) for providing F2 and F3/C satellites data. The authors would also like to thank Dr. Shiann-Jeng Yu, NSPO Deputy Director General, for his useful suggestions. This research work is partially supported by the National Science Council with grant NSC102-2628-M-008-001.

\section{REFERENCES}

Abdu, M. A., I. S. Batista, A. J. Carrasco, and C. G. M. Brum,
2005: South Atlantic magnetic anomaly ionization: A review and a new focus on electrodynamic effects in the equatorial ionosphere. J. Atmos. Sol.-Terr. Phys., 67, 1643-1657, doi: 10.1016/j.jastp.2005.01.014. [Link]

Baker, D. N., 2000: The occurrence of operational anomalies in spacecraft and their relationship to space weather. IEEE T. Plasma Sci., 28, 2007-2016, doi: 10.1109/27.902228. [Link]

Bashkirov, V. F., N. V. Kuznetsov, and R. A. Nymmik., 1999: An analysis of the SEU rate of microcircuits exposed by the various components of space radiation. Radiat. Meas., 30, 427-433.

Campbell, A., P. McDonald, and K. Ray, 1992: Single event upset rates in space. IEEE T. Nucl. Sci., 39, 1828-1835, doi: 10.1109/23.211373 . [Link]

Coker, C., K. F. Dymond, S. A. Budzien, D. H. Chua, J. Y. Liu, D. N. Anderson, S. Basu, and T. R. Pedersen, 2009: Observations of the ionosphere using the Tiny ionospheric Photometer. Terr. Atmos. Ocean. Sci., 20, $227-$ 235, doi: 10.3319/TAO.2008.01.18.02(F3C). [Link]

Dessler, A. J., 1959: Effect of magnetic anomaly on particle 
radiation trapped in geomagnetic field.J.Geophys. Res., 64, 713-715, doi: 10.1029/JZ064i007p00713. [Link]

Fleetwood, D. M., P. S. Winokur, and P. E. Dodd, 2000: An overview of radiation effects on electronics in the space telecommunications environment. Microelectron. Reliab., 40, 17-26, doi: 10.1016/S0026-2714(99)00225-5. [Link]

Fong, C. J., N. L. Yen, C. H. Chu, S. K. Yang, W. T. Shiau, C. Y. Huang, S. Chi, S. S. Chen, Y. A. Liou, and Y. H. Kuo, 2009: FORMOSAT-3/COSMIC spacecraft constellation system, mission results, and prospect for follow-on mission. Terr. Atmos. Ocean. Sci., 20, 1-19, doi: 10.3319/TAO.2008.01.03.01(F3C). [Link]

Gledhill, J. A., 1976: Aeronomic effects of the South Atlantic anomaly. Rev. Geophys. Space Phys., 14, 173-187, doi: 10.1029/RG014i002p00173. [Link]

Gubby, R. and J. Evans, 2002: Space environment effects and satellite design. J. Atmos. Sol.-Terr. Phys., 64, 17231733, doi: 10.1016/S1364-6826(02)00122-0. [Link]

Guenzer, C. S., E. A. Wolicki, and R. G. Allas, 1979: Single event upset of dynamic RAMs by neutrons and protons. IEEE T. Nucl. Sci., 26, 5048-5052, doi: 10.1109/ TNS.1979.4330270 [Link]

Hsu, M. L., P. K. Rajesh, J. Y. Liu, L. C. Tsai, H. F. Tsai, C. H. Lin, K. F. Dymond, C. Coker, D. H. Chua, S. A. Budzien, and C. Z. Cheng, 2009: Ionospheric electron density concurrently derived by TIP and GOX of FORMOSAT-3/COSMIC. Terr. Atmos. Ocean. Sci., 20, 207-214, doi: 10.3319/TAO.2008.04.24.02(F3C). [Link]

Iucci, N., L. I. Dorman, A. E. Levitin, A. V. Belov, E. A. Eroshenko, N. G. Ptitsyna, G. Villoresi, G. V. Chizhenkov, L. I. Gromova, M. Parisi, M. I. Tyasto, and V. G. Yanke, 2006: Spacecraft operational anomalies and space weather impact hazards. Adv. Space Res., 37, 184-190, doi: 10.1016/j.asr.2005.03.028. [Link]

Kolasinski, W. A., J. B. Blake, J. K. Anthony, W. E. Price, and E. C. Smith, 1979: Simulation of cosmic-ray induced soft errors and latchup in integrated-circuit computer memories. IEEE T. Nucl. Sci., 26, 5087-5091, doi: 10.1109/TNS.1979.4330278. [Link]

Koshiishi, H., H. Matsumoto, and T. Goka, 2008: Singleevent upset in geostationary transfer orbit during solaractivity maximum period measured by the Tsubasa satellite. Adv. Space Res., 42, 1500-1503, doi: 10.1016/j. asr.2007.11.026. [Link]

Kuznetsov, N. V., N. I. Nikolaeva, and. M. I. Panasyuk, 2010: Variation of the trapped proton flux in the inner radiation belt of the earth as a function of solar activity. Cosmic Res., 48, 80-85, doi: 10.1134/ S0010952510010065. [Link]

Liou, Y. A., A. G. Pavelyev, S. F. Liu, , A. A. Pavelyev, N. Yen, , C. Y. Huang, and C. J. Fong, 2007: FOR-
MOSAT-3/COSMIC GPS radio occultation mission: Preliminary results. IEEE Trans. Geosci. Remote Sensing, 45, 3813-3826, doi: 10.1109/TGRS.2007.903365. [Link]

Liu, J. Y., Y. I. Chen, Y. J. Chuo, and C. S. Chen, 2006: A statistical investigation of preearthquake ionospheric anomaly. J. Geophys. Res., 111, A05304, doi: 10.1029/2005JA011333. [Link]

Lyons, L. R., 1997: Magnetospheric processes leading to precipitation. Space Sci. Rev., 80, 109-132, doi: 10.1023/A:1004977704864. [Link]

Nee, J. B., S. D. Tsai, T. H. Peng, R. R. Hsu, A. B. C. Chen, S. Zhang, T. Y. Huang, P. K. Rajesh, J. Y. Liu, H. U. Frey, and S. B. Mende, 2010: OH airglow and equatorial variations observed by ISUAL instrument on board the FORMOSAT 2 satellite. Terr. Atmos. Ocean. Sci., 21, 985-995, doi: 10.3319/TAO.2010.03.12.01(AA). [Link]

Nichitiu, F., J. R. Drummond, J. Zou, and R. Deschambault, 2004: Solar particle events seen by the MOPITT instrument. J. Atmos. Sol.-Terr. Phys., 66, 1797-1803, doi: $10.1016 /$ j.jastp.2004.06.002. [Link]

Patil, C. G., G. Rajaram, and M. Y. S. Prasad, 2008: Correlation of GSO satellite anomalies with space weather data. Acta Astronautica, 63, 458-470, doi: 10.1016/j. actaastro.2007.12.050. [Link]

Rajesh, P. K., J. Y. Liu, C. Y. Chiang, A. B. Chen, W. S. Chen, H. T. Su, R. R. Hsu, C. H. Lin, M. L. Hsu, J. H. Yee, and J. B. Nee, 2009: First results of the limb imaging of $630.0 \mathrm{~nm}$ airglow using FORMOSAT-2/Imager of Sprites and Upper Atmospheric Lightnings. J. Geophys. Res., 114, A10302, doi: 10.1029/2009JA014087. [Link]

Reed, E. I., W. B. Fowler, and J. E. Blamont, 1973: An atlas of low-latitude 6300-A [O I] night airglow from Ogo 4 observations. J. Geophys. Res., 78, 5658-5675, doi: 10.1029/JA078i025p05658. [Link]

Rocken, C., Y. H. Kuo, W. S. Schreiner, D. Hunt, S. Sokolovskiy, and C. McCormick, 2000: COSMIC System Description. Terr. Atmos. Ocean. Sci., 11, 21-52.

Sergeev, V. A., E. M. Sazhina, N. A. Tsyganenko, J. Å. Lundblad, and F. Søraas, 1983: Pitch-angle scattering of energetic protons in the magnetotail current sheet as the dominant source of their isotropic precipitation into the nightside ionosphere. Planet. Space Sci., 31, 11471155, doi: 10.1016/0032-0633(83)90103-4. [Link]

Torr, D. G., M. R. Torr, J. C. G. Walker, and R. A. Hoffman, 1975: Particle precipitation in the south atlantic geomagnetic anomaly. Planet. Space Sci., 23, 15-26, doi: 10.1016/0032-0633(75)90064-1. [Link]

Vernov, S. N., E. V. Gorchakov, P. I. Shavrin, and K. N. Sharvina, 1967: Radiation belts in the region of the South-Atlantic magnetic anomaly. Space Sci. Rev., 7, 490-533, doi: 10.1007/BF00182684. [Link] 